\title{
Noninvasive Ventilation for Patients With Acute Lung Injury or Acute Respiratory Distress Syndrome
}

\author{
Stefano Nava MD, Ania Schreiber MD, and Guido Domenighetti MD

\begin{abstract}
Introduction
Physiological Rationale

Meta-analyses and Systematic Reviews

NIV to Prevent Endotracheal Intubation in ALI/ARDS Patients

NIV as an Alternative to Endotracheal Intubation in ALI/ARDS Patients

Summary
\end{abstract}

\begin{abstract}
Few studies have been performed on noninvasive ventilation (NIV) to treat hypoxic acute respiratory failure in patients with acute lung injury (ALI) or acute respiratory distress syndrome (ARDS). The outcomes of these patients, for whom endotracheal intubation is not mandatory, depend on the degree of hypoxia, the presence of comorbidities and complications, and their illness severity. The use of NIV as an alternative to invasive ventilation in severely hypoxemic patients with ARDS (ie, $\mathrm{P}_{\mathrm{aO}} / \mathrm{F}_{\mathrm{IO}_{2}}<200$ ) is not generally advisable and should be limited to hemodynamically stable patients who can be closely monitored in an intensive care unit by highly skilled staff. Early NIV application may be extremely helpful in immunocompromised patients with pulmonary infiltrates, in whom intubation dramatically increases the risk of infection, pneumonia, and death. The use of NIV in patients with severe acute respiratory syndrome and other airborne diseases has generated debate, despite encouraging clinical results, mainly because of safety issues. Overall, the high rate of NIV failure suggests a cautious approach to NIV use in patients with ALI/ARDS, including early initiation, intensive monitoring, and prompt intubation if signs of NIV failure emerge. Key words: noninvasive ventilation; NIV; acute respiratory distress syndrome; ARDS; acute lung injury; ALI; intubation. [Respir Care 2011;56(10):1583-1588. (C) 2011 Daedalus Enterprises]
\end{abstract}

\section{Introduction}

The application of noninvasive ventilation (NIV) in patients with severe hypoxemic acute respiratory failure

Dr Nava is affiliated with Pneumologia e Terapia Intensiva Respiratoria, Dipartimento Cardio-Toraco-Vascolare, Policlinico Sant' OrsolaMalpighi, Bologna, Italy. Dr Schreiber is affiliated with the Respiratory Unit, Fondazione S Maugeri, Istituto di Ricovero e Cura a Carattere Scientifico, Pavia, Italy. Dr Domenighetti is affiliated with the Intensive Care Unit, Ospedale della Carita', Locarno, Switzerland.

Dr Nava presented a version of this paper at the 26th New Horizons Symposium, "ARDS Update," at the 56th International Respiratory Congress of the American Association for Respiratory Care, held December 6-9, 2010, in Las Vegas, Nevada.

The authors have disclosed no conflicts of interest.
(ARF) is controversial, despite some promising results. ${ }^{1-4}$ So far, published clinical randomized and non-randomized studies on NIV in non-COPD-related hypoxemic ARF have been mainly focused on single etiologies (cardiogenic pulmonary edema, community-acquired pneumonia, or both $^{2-7}$ ) or on mixed populations with various diagnoses. ${ }^{1,8,9}$ In the latter studies, the clinical outcome according to the underlying nature of hypoxemic ARF was seldom assessed. ${ }^{8,9}$ However, and interestingly enough, some of the

\footnotetext{
Correspondence: Stefano Nava MD, Pneumologia e Terapia Intensiva Respiratoria, Policlinico Sant Orsola-Malpighi, via Massarenti n.9, Bologna 40138 Italy. E-mail: stefano.nava@aosp.bo.it.
}

DOI: $10.4187 /$ respcare.01209 
latter investigations, as well as others, ${ }^{9,10}$ included noninvasively ventilated patients who had a diagnosis of classical ARDS. ${ }^{11}$ The application of NIV in hypoxemic ARF is, however, still controversial. An International Consensus Conference on NIV 12 stated that "larger, controlled studies are required to determine the potential benefit of adding NIV to standard medical treatment in the avoidance of endotracheal intubation."

The definition and severity of an episode of hypoxemic respiratory failure still relies on $\mathrm{P}_{\mathrm{aO}_{2}} / \mathrm{F}_{\mathrm{IO}_{2}}$, which includes various conditions of different etiology under the same umbrella. Hypoxemic ARF can be the "end point" of several pathologies, each acting through different pathophysiological mechanisms (shunt, ventilation-perfusion mismatch, impairment of alveolar-capillary diffusion), or to the pathogenesis of the disease (primary pulmonary or extrapulmonary ARDS), and it is difficult to draw conclusions about the efficacy of a treatment when the underlying disease is not considered as a confounder in the statistical analysis.

Acute lung injury (ALI) and its more severe form, acute respiratory distress syndrome (ARDS), are common and devastating complications during acute illness and after injury, and ALI and ARDS have high morbidity and mortality and long-term decrease in quality of life. ${ }^{13}$

Among other strategies aimed to improve the early diagnosis and/or to prevent ALI/ARDS progression, NIV has been advocated to avoid invasive ventilation and its infectious pulmonary and non-pulmonary complications. ${ }^{14}$ However, the benefits of NIV in these critically ill patients must be balanced against the potential harms of NIV failure and inappropriate delay of intubation, which increases the risk of death. ${ }^{15}$

Despite there having been very few studies, and those mainly observational, we will review the role of NIV in ALI/ARDS patients and highlight the timing of NIV application (ie, NIV to avoid intubation or as an alternative to intubation). Most of the studies in the literature described the use of NIV in hypoxemic ARF, and considered ALI and ARDS almost as a unique entity, so we will also use a similar approach, while recognizing that in reality ALI encompasses ARDS. ${ }^{11}$

\section{Physiological Rationale}

From a physiological point of view, the application of positive airway pressure opens under-ventilated alveoli and increases functional residual capacity, thus decreasing right-to-left intrapulmonary shunt and improving lung mechanics. ${ }^{16}$ In patients with ALI, NIV is proven to increase oxygenation, reduce dyspnea, and unload respiratory muscles. ${ }^{17}$. In those patients, continuous positive airway pressure alone improves gas exchange but does not unload the respiratory muscles, whereas NIV provides a better re- sponse, unloading the muscles and relieving dyspnea, compared to continuous positive airway pressure. ${ }^{17}$ By lowering left-ventricular transmural pressure in patients with congestive left-heart failure, positive airway pressure may reduce left-ventricular afterload without compromising cardiac index. ${ }^{18,19}$

\section{Meta-analyses and Systematic Reviews}

A systematic review suggested that the addition of NIV to standard medical therapy in patients with hypoxemic ARF reduces the intubation rate, shortens intensive care unit (ICU) stay, and lowers ICU mortality. ${ }^{20}$ However, because of substantial patient heterogeneity in the included studies, that result cannot be extrapolated to the subset of patients with ALI and ARDS. Agarwal et $\mathrm{al}^{21}$ conducted a meta-analysis of randomized controlled trials and included only patients with ALI/ARDS. The results suggested that patients with ALI/ARDS were unlikely to have important outcome benefits from NIV added to standard therapy, but the analysis included only 3 studies (with a total of 111 patients), so there was a chance of type I and type II errors. Very recently Agarwal et $a^{22}$ conducted another metaanalysis in which they included 13 studies (with 540 patients). The intubation rate ranged from $30 \%$ to $86 \%$, and the pooled intubation rate was $48 \%$ (95\% CI 39-58\%). The mortality rate ranged from $15 \%$ to $71 \%$, and the pooled mortality rate was 35\% (95\% CI 26-45\%). There was statistically significant heterogeneity in intubation rate or mortality, and no evidence of publication bias. However, with few exceptions, the studies analyzed were not randomized, and, most important, the patients had heterogeneous underlying pathologies (eg, community-acquired pneumonia, sepsis, and near-drowning), which disallows firm conclusions. An accompanying editorial ${ }^{23}$ stated that the conclusions we can draw from that meta-analysis are limited and that we desperately need a randomized trial on the risks and benefits of NIV in ALI. So, to summarize a recent evidence-based review, ${ }^{24}$ the evidence points to extreme caution in the use NIV in ARDS, and this recommendation is obtained from systematic reviews (with homogeneity) of case-control studies.

\section{NIV to Prevent Endotracheal Intubation in ALI/ARDS Patients}

Few clinical trials have evaluated NIV as a means to prevent intubation in patients with mild to moderate hypoxemic ARF (ie, $\mathrm{P}_{\mathrm{aO}_{2}} / \mathrm{F}_{\mathrm{IO}_{2}} \sim 200$ ) of various etiologies. The results have been controversial. Most of the studies enrolled patients who did not have indications that would mandate immediate endotracheal intubation under today's standard of care. 
Rocker et al ${ }^{10}$ reported on 12 episodes of ALI/ARDS in 10 patients treated with NIV. The ARDS etiologies were malaria, fat emboli, postpartum thrombotic microangiopathy, inhalation injury, near-drowning, and pneumonia. Rocker et al did not mention how many ARDS patients they cared for in their ICU who were not included. NIV succeeded (intubation avoided and no further assisted ventilation for 72 hours) on 6 occasions, and 7 of the 10 patients survived hospitalization. Rocker et al concluded that in hemodynamically stable patients with severe ALI/ ARDS, NIV had a high success rate, and NIV should be considered in stable patients who are in the early phase of ALI/ARDS.

Ferrer et $\mathrm{al}^{9}$ randomized 105 patients with severe hypoxemic ARF to receive, via oronasal mask, either NIV or high $\mathrm{F}_{\mathrm{IO}_{2}}$. The respiratory-failure etiologies were mainly pneumonia and cardiogenic lung edema, but there were 15 patients with ARDS: 7 in the NIV group, and 8 in the control group. Ferrer et al did not differentiate ARDS of primary versus secondary origin. The efficacy of NIV was poor in the ARDS subgroup, and the multivariate statistical analysis aimed to identify the factors associated with NIV failure showed a very high relative risk for the patients with ARDS.

The role of NIV in the treatment of ALI was assessed in critically ill patients with bilateral infiltrates of different origin in a study from the Mayo Clinic. ${ }^{25}$ In this observational cohort study, Rana and co-workers prospectively assessed the outcomes of 54 consecutive patients and tried to identify specific risk factors for the NIV failure. ${ }^{17}$ Causes of ALI were pneumonia, nonpulmonary sepsis, vasculitis, and exacerbation of interstitial lung disease. They found a high failure rate and a higher than expected mortality, and concluded that, unless the underlying shock, metabolic acidosis, and severe hypoxemia are not rapidly resolved, the rate of NIV failure is very high and that a "judicious" trial of NIV should be instituted only with extreme caution in these patients. NIV success was significantly correlated with lower mean illness severity scores (Acute Physiology and Chronic Health Evaluation III or Sequential Organ Failure Assessment), with higher admission $\mathrm{P}_{\mathrm{aO}_{2}} / \mathrm{F}_{\mathrm{IO}_{2}}$ (147 vs 112), and with less pronounced acidosis.

Yoshida et $a^{26}$ retrospectively studied 47 consecutive patients with a diagnosis of ALI of pulmonary and extrapulmonary origin. Thirty-three patients successfully avoided intubation. Patients who required intubation had a significantly higher Acute Physiology and Chronic Health Evaluation II score $(>17)$ and a significantly lower arterial $\mathrm{pH}$. Respiratory rate decreased significantly within one hour of starting NIV only in the patients successfully treated with NIV. Patients who avoided intubation had a significantly lower ICU mortality rate and hospital mortality rate than patients who required intubation.
In another observational study, matched with a control group, Domenighetti and co-workers ${ }^{27}$ considered an ARDS group of 12 patients without distant organ failure at admission, and with a direct pulmonary ARDS due to pneumonia, near-drowning, or toxic agents. They found that NIV might be feasible in stable non-immunocompromised patients who meet the definition of a primary ARDS and present at baseline or during the course of the disease without extrapulmonary organ dysfunction. Not surprisingly, avoidance of intubation was associated with faster weaning and fewer complications. By analyzing possible predictors of NIV failure, Domenighetti et al found that all patients who needed intubation progressed to sepsis (positive blood cultures) and eventually to multiple-organ dysfunction, contrasting with the absence of bacteremia and extrapulmonary organ failure in patients who successfully responded to NIV.

Agarwal et al,28 in prospective observational study over a one-and-a-half-year period, enrolled consecutive patients ventilated for hypoxic respiratory failure. The patients were classified into 2 groups, based on the etiology of respiratory failure: ALI/ARDS, and other causes. During the study period, 287 patients were admitted in the ICU, and of the 40 who were initiated on NIV, 21 had ALI/ARDS. The NIV failures, the mean ICU and hospital stay, and the hospital mortality were similar in the 2 groups; however, 12 of the 21 ALI-ARDS patients needed intubation, versus 7 of the 19 in the other group. In the univariate logistic regression model the only factor associated with NIV failure was the baseline $\mathrm{P}_{\mathrm{aO}_{2}} / \mathrm{F}_{\mathrm{IO}_{2}}$ (odds ratio $0.97,95 \% \mathrm{CI}$ 0.95-0.99).

Guisset et al$^{29}$ reported the results of their randomized controlled trial in abstract form. Eighty-four patients were randomized to receive NIV or standard medical therapy at the early onset of hypoxemic ARF due to ARDS. The NIV group had a lower intubation rate and lower ICU and hospital mortality. These data should be considered with caution, while waiting for publication in a peer-reviewed journal.

The 2 most important studies that distinguished primary and secondary ARDS in addressing NIV in patients with hypoxic ARF were both by Antonelli et al. ${ }^{8,30}$ In their first prospective multicenter cohort study, they investigated factors involved in failure. In a heterogeneous population, the overall efficacy of NIV in avoiding intubation (70\%) contrasted with the highest rate of failure observed in 86 patients fulfilling the diagnosis of ARDS (51\%). The intubation rate was similar among patients with ARDS of pulmonary versus extrapulmonary origin, but sepsis on admission was associated, among other variables, with NIV failure. $^{8}$

A later study ${ }^{30}$ performed in 3 European ICUs with NIV expertise clarified the "real life" use of NIV in ARDS patients. "Only" $17 \%$ of the patients admitted with ARDS 
were successfully treated with NIV. Over 2 years, 479 patients were admitted to the 3 ICUs with hypoxemic ARF due to ARDS. Three-hundred thirty-two (69\%) of those patients were already intubated at admission, so 147 were eligible for the study. NIV improved gas exchange and avoided intubation in 79 (54\%) of those 147 patients, leading to an overall NIV success rate of $<20 \%$. This was associated with less ventilator-associated pneumonia and a lower ICU mortality rate (6\% vs $53 \%$ ). The response to NIV was similar in patients with ARDS of pulmonary versus extrapulmonary origin. Endotracheal intubation was more likely in patients with higher Simplified Acute Physiology Score (SAPS II) and who needed a higher level of inspiratory support. A SAPS II $>34$ and inability to improve $\mathrm{P}_{\mathrm{aO}_{2}} / \mathrm{F}_{\mathrm{IO}_{2}}$ after one hour of NIV were predictors of failure. In summary, the use of NIV as an alternative to invasive ventilation in severely hypoxemic patients with ARDS (ie, $\mathrm{P}_{\mathrm{aO}_{2}} / \mathrm{F}_{\mathrm{IO}_{2}}<200$ ) is not generally advisable and should be limited to hemodynamically stable patients who can be closely monitored in an ICU by highly skilled staff.

Early NIV application may be extremely helpful in immunocompromised patients with pulmonary infiltrates not necessarily associated with ALI, in whom intubation dramatically increases the risk of pneumonia, infection, and ICU mortality. Two trials evaluated NIV versus standard treatment alone in immunocompromised patients with respiratory rate $>30$ breaths $/ \mathrm{min}$ and $\mathrm{P}_{\mathrm{aO}_{2}} / \mathrm{F}_{\mathrm{IO}_{2}}$ of approximately 200. Antonelli et $\mathrm{al}^{4}$ compared NIV versus standard therapy in solid-organ-transplant recipients with hypoxemic ARF. Within the first hour of treatment, $\mathrm{P}_{\mathrm{aO}_{2}}$ ' $\mathrm{F}_{\mathrm{IO}_{2}}$ improved in $70 \%$ of the NIV group and only $25 \%$ of the control group. NIV was associated with significantly less intubation, fewer complications, lower mortality, and shorter ICU stay among survivors. In patients with immunosuppression secondary to hematological malignancies, transplantation, or human immunodeficiency virus infection, Hilbert et $\mathrm{al}^{3}$ compared early NIV to standard treatment. All the patients had fever, bilateral pulmonary infiltrates, and hypoxemia. Fewer patients in the NIV group required intubation, had serious complications, or died in the ICU or hospital. The latter 2 studies are now both about 10 years old. This is relevant because later studies ${ }^{31}$ on the outcomes of these patients suggest that, if intubated, they do better than in the past, so that the impressive results obtained in the late 1990s with NIV in this specific population may now also be obtained with invasive ventilation.

The use of NIV for severe acute respiratory syndrome (SARS) and other airborne diseases has generated debate. Based on the Toronto experience with SARS, in which some clinicians contracted SARS when a patient was intubated following NIV failure, the use of NIV in these patients was discouraged. ${ }^{32}$ Two subsequent observational studies from China, ${ }^{33,34}$ however, found no evidence of viral spread to clinicians who took appropriate precautions. In the event of a bird flu pandemic, ventilator resources are likely to be severely strained, and NIV may offer a means of supporting some of the afflicted, mainly those with initial respiratory failure. However, some consider NIV contraindicated in respiratory failure from airborne respiratory diseases unless it is used in a negativepressure isolation room and strict precautions are taken.

In conclusion, the outcome of NIV in patients with hypoxemic ARF due to ALI, ARDS, or severe pulmonary infiltrates, and for whom endotracheal intubation is not mandatory, depends on the degree of hypoxia, the presence of comorbidities and complications, and the illness severity scores. The high rate of NIV failure suggests a cautious approach with these patients, consisting of early NIV trial and no delay of needed intubation. A trial of NIV is advisable in immunosuppressed patients, in whom intubation is a strong predictor of mortality.

Overall, when using NIV in a patient with hypoxic ARF, in an attempt to avoid intubation, one should always consider the risks of inappropriate intubation delay. Demoule et $\mathrm{al}^{15}$ found that the effect of NIV differs between de novo hypoxemic ARF (including mainly in this definition ALI and ARDS) and patients with cardiogenic pulmonary edema or COPD, because NIV failure is associated with higher mortality in patients with de novo hypoxemic ARF. This finding should therefore raise a note of caution when applying NIV for this indication

\section{NIV as an Alternative to Endotracheal Intubation in ALI/ARDS Patients}

To our knowledge, only one randomized trial has evaluated the use of NIV in hypoxemic patients considered sufficiently ill to require mechanical ventilation. Antonelli et al $^{1}$ compared NIV to conventional ventilation via endotracheal tube in selected patients with hypoxemic ARF secondary to cardiogenic pulmonary edema, ALI/ARDS, or pneumonia, who failed to improve despite aggressive medical therapy and who met predefined criteria for mechanical ventilation. Sixty-four consecutive patients were enrolled (32 in each arm). After one hour of mechanical ventilation, $\mathrm{P}_{\mathrm{aO}_{2}} / \mathrm{F}_{\mathrm{IO}_{2}}$ had improved in both groups. Ten patients in the NIV group required intubation. The conventional-ventilation patients more frequently developed serious complications ( $66 \%$ vs $38 \%$,) and, in particular, infection secondary to intubation (ie, pneumonia and/or sinusitis). Among survivors, the duration of ventilation and ICU stay was shorter in the NIV patients. Seven of the 32 NIV patients were affected by ARDS, versus 9 of the 32 conventional-ventilation patients, but no subgroup analysis was performed. Bear in mind, however, that this study included a selected small group of patients in a highly experienced center and a heterogeneous population. 


\section{NIV FOR ALI OR ARDS}

\section{Summary}

Few data support routine use of NIV in the treatment of hypoxic ARF in ALI/ARDS patients. Most of the available studies suggest that NIV should be avoided as a first ventilatory support in ARDS patients who have a clinical picture of extrapulmonary organ dysfunction at admission or in those developing a distant organ failure during the course of the disease (ie, mainly established septic patients). Moreover, NIV should be avoided unless the underlying shock, metabolic acidosis, and severe hypoxemia $\left(\mathrm{P}_{\mathrm{aO}} / \mathrm{F}_{\mathrm{IO}_{2}}<150\right)$ are rapidly resolved. Older age, higher SAPS II score at admission, and lower $\mathrm{P}_{\mathrm{aO}_{2}} / \mathrm{F}_{\mathrm{IO}_{2}} \leq 175$ ) at one hour of NIV predict intubation in these patients. However, some encouraging results, though with the abovedescribed limitations, suggest that in stable homogeneous patients with ALI-ARDS, NIV merits further randomized studies. Timing of application is also important, because early NIV application to avoid intubation in less hypoxic patients $\left(\mathrm{P}_{\mathrm{aO}_{2}} / \mathrm{F}_{\mathrm{IO}_{2}}>200\right)$ may be useful. Until additional data are available, we recommend a conservative approach (eg, a brief NIV trial in a less severely ill patient), particularly in centers that are not expert in NIV. Further multicenter randomized controlled trials are needed to assess the efficacy of NIV to avoid intubation and as an alternative to it, and to better understand the subset of patients most likely to benefit.

\section{REFERENCES}

1. Antonelli M, Conti G, Rocco M, Bufi M, De Blasi RA, Vivino G, et al. A comparison of noninvasive positive-pressure ventilation and conventional mechanical ventilation in patients with acute respiratory failure. N Engl J Med 1998;339(7):429-435.

2. Confalonieri M, Potena A, Carbone G, Porta RD, Tolley EA, Meduri GU. Acute respiratory failure in patients with severe communityacquired pneumonia: a prospective randomized evaluation of noninvasive ventilation. Am J Respir Crit Care Med 1999;160(5):15851591.

3. Hilbert G, Gruson D, Vargas F, Valentino R, Gbikpi-Benissan G, Dupon M, et al. Noninvasive ventilation in immunosuppressed patients with pulmonary infiltrates, fever, and acute respiratory failure. N Engl J Med 2001;344(7):481-487.

4. Antonelli M, Conti G, Bufi M, Costa MG, Lappa A, Rocco M, et al. Noninvasive ventilation for treatment of acute respiratory failure in patients undergoing solid organ transplantation: a randomized trial. JAMA 2000;283(2):235-241.

5. Masip J, Betbese AJ, Paez J, Vecilla F, Canizares R, Padro J, et al. Noninvasive pressure support ventilation versus conventional oxygen therapy in acute cardiogenic pulmonary edema: a randomized trial. Lancet 2000;356(9248):2126-2132.

6. Domenighetti G, Gayer R, Gentilini R. Noninvasive pressure support ventilation in non-COPD patients with acute cardiogenic pulmonary edema and severe community-acquired pneumonia: acute effects and outcome. Intensive Care Med 2002;28(9):1226-1232.

7. Jolliet P, Abajo B, Pasquina P, Chevrolet JC. Noninvasive pressure support ventilation in severe community-acquired pneumonia. Intensive Care Med 2001;27(5):812-821.
8. Antonelli M, Conti G, Moro ML, Esquinas A, Gonzalez-Diaz G, Confalonieri M, et al. Predictors of failure of noninvasive positive pressure ventilation in patients with acute hypoxemic respiratory failure: a multi-center study. Intensive Care Med 2001;27(11):17181728.

9. Ferrer M, Esquinas A, Leon M, Gonzalez G, Alarcon A, Torres A. Noninvasive ventilation in severe hypoxemic respiratory failure. A randomized clinical trial. Am J Respir Crit Care Med 2003;168(12): 1438-1444.

10. Rocker GM, Mackenzie MG, Williams B, Logan PM. Noninvasive pressure support ventilation: successful outcome in patients with acute lung injury/ARDS. Chest 1999;115(1):173-177.

11. Bernard GR, Artigas A, Brigham KL, Carlet J, Falke K, Hudson L, et al. Report of the American-European consensus conference on ARDS: definitions, mechanisms, relevant outcomes and clinical trial coordination. Intensive Care Med 1994;20(3):225-232.

12. International Consensus Conferences in Intensive Care Medicine: noninvasive positive pressure ventilation in acute respiratory failure. Organised jointly by the American Thoracic Society; European Respiratory Society; European Society of Intensive Care Medicine; Société de Réanimation de Langue Française, and approved by the ATS Board of Directors December 2000. Intensive Care Med 2001; 27(1):166-178.

13. Hopkins RO, Weaver LK, Collingridge D, Parkinson RB, Chan KJ, Orme F Jr. Two-year cognitive, emotional, and quality-of-life outcomes in acute respiratory distress syndrome. Am J Respir Crit Care Med 2005;2005;171(3):340-347

14. Girou E, Brun-Buisson C, Taillé S, Lemaire F, Brochard L. Secular trends in nosocomial infections and mortality associated with noninvasive ventilation in patients with exacerbation of COPD and pulmonary edema. JAMA 2003;290(22):2985-2991.

15. Demoule A, Girou E, Richard JC, Taille S, Brochard L. Benefits and risks of success or failure of noninvasive ventilation. Intensive Care Med 2006;32(11):1756-1765.

16. Katz JA, Marks JD. Inspiratory work with and without continuous positive airway pressure in patients with acute respiratory failure. Anesthesiology 1985;63(6):598-607.

17. L'Her E, Deye N, Lellouche F, Taille S, Demoule A, Fraticelli A, et al. Physiologic effects of noninvasive ventilation during acute lung injury. Am J Respir Crit Care Med 2005;172(9):1112-1118.

18. Räsänen J, Heikkilä J, Downs J, Nikki P, Väisänen I, Viitanen. A. Continuous positive airway pressure by face mask in acute cardiogenic pulmonary edema. Am J Cardiol 1985;55(4):296-300.

19. Naughton MT, Rahman MA, Hara K, Floras S, Bradley TD. Effect of continuous positive airway pressure on intrathoracic and left ventricular transmural pressures in patients with congestive heart failure. Circulation 1995;91(6):1725-1731.

20. Keenan SP, Sinuff T, Cook DJ, Hill NS. Does noninvasive positive pressure ventilation improve outcome in acute hypoxemic respiratory failure? A systematic review. Crit Care Med 2004;32(12):25162523.

21. Agarwal R, Aggarwal AN, Gupta D. Is there a role for noninvasive ventilation in acute respiratory distress syndrome? A meta-analysis. Respir Med 2006;100(12):2235-2238.

22. Agarwal R, Aggarwal AN, Gupta D. Role of noninvasive ventilation in acute lung injury/acute respiratory distress syndrome: a proportion meta-analysis. Respir Care 2010;55(12):1653-1660.

23. Keenan SP. Noninvasive ventilation in acute lung injury: caution is good, more evidence is better (editorial). Respir Care 2010;55(12): 1757-1758.

24. Nava S, Hill N. Non-invasive ventilation in acute respiratory failure. Lancet 2009;374(9685):250-259. 


\section{NIV FOR ALI OR ARDS}

25. Rana S, Jenad H, Gay P, Buck C, Hubmayr R, Gajic O. Failure of non-invasive ventilation in patients with acute lung injury: observational cohort study. Critical Care 2006;10:R79 DOI: 10.1186/cc4923

26. Yoshida Y, Takeda S, Akada S, Hongo T, Tanaka K, Sakamoto A. Factors predicting successful noninvasive ventilation in acute lung injury. J Anesth 2008;22(3):201-206.

27. Domenighetti G, Moccia A, Gayer R. Noninvasive ventilation in patients with a primary acute respiratory distress syndrome. An observational case-control study in a homogeneous group of patients. Monaldi Arch Chest Dis 2008;69(1):5-10.

28. Agarwal R, Handa A, Aggarwal AN, Gupta D, Behera D. Outcomes of noninvasive ventilation in acute hypoxemic respiratory failure in a respiratory intensive care unit in north India. Respir Care 2009; 54(12):1679-1687.

29. Guisset O, Gruson D, Vargas F, Gabinski C, Gbikpi-Benissan G, Guenard H, Hilbert G. Noninvasive ventilation in acute respiratory distress syndrome (ARDS) patients (abstract). Intensive Care Med 2003;29(Suppl):S124.
30. Antonelli M, Conti G, Esquinas A, Montini L, Maggiore SM, Bello $\mathrm{G}$ et al. A multiple-center survey on the use in clinical practice of noninvasive ventilation as a first-line intervention for acute respiratory distress syndrome. Crit Care Med 2007;35(1):18-25.

31. Pipeling MR, Fan E. Therapies for refractory hypoxemia in acute respiratory distress syndrome. JAMA 2010;304(22):2521-2527.

32. Poutanen SM, Low DE, Henry B, Finkelstein S, Rose D, Green K, et al. Identification of severe acute respiratory syndrome in Canada. N Engl J Med 2003;348(20):1195-2005.

33. Cheung TMT, Lau CWA, Poon E, So, Kong B, Yung R. Effectiveness of noninvasive positive pressure ventilation in the treatment of acute respiratory failure in severe acute respiratory syndrome. Chest 2004;126(3):845-850.

34. Zaho Z, Zhang F, Xu M, Huang K, Zhong W, Cai W, et al. Description and clinical treatment of an early outbreak of severe acute respiratory syndrome (SARS) in Guangzhou, PR China. J Med Microbiol 2003;52(8):715-720. 